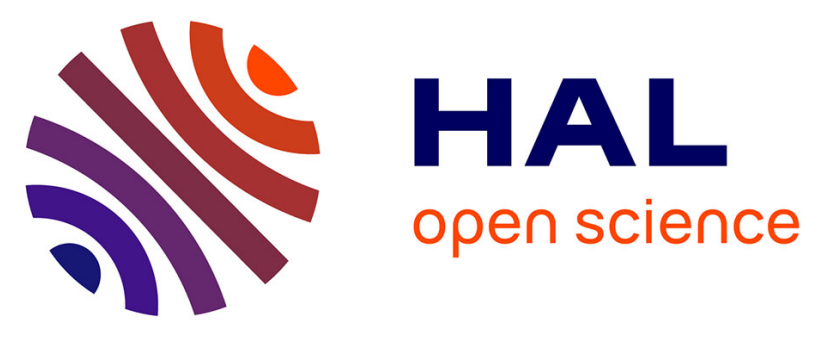

\title{
Assembly of microscopic highly magnetic droplets: Magnetic alignment versus viscous drag
}

Olivier Sandre, Julien Browaeys, Régine Perzynski, Jean-Claude Bacri, Valérie Cabuil, Ronald E. Rosensweig

\section{- To cite this version:}

Olivier Sandre, Julien Browaeys, Régine Perzynski, Jean-Claude Bacri, Valérie Cabuil, et al.. Assembly of microscopic highly magnetic droplets: Magnetic alignment versus viscous drag. Physical Review E , 1999, 59 (2), pp.1736-1746. 10.1103/PhysRevE.59.1736 . hal-02168821

\section{HAL Id: hal-02168821 \\ https://hal.science/hal-02168821}

Submitted on 25 Dec 2019

HAL is a multi-disciplinary open access archive for the deposit and dissemination of scientific research documents, whether they are published or not. The documents may come from teaching and research institutions in France or abroad, or from public or private research centers.
L'archive ouverte pluridisciplinaire HAL, est destinée au dépôt et à la diffusion de documents scientifiques de niveau recherche, publiés ou non, émanant des établissements d'enseignement et de recherche français ou étrangers, des laboratoires publics ou privés. 


\title{
Assembly of Microscopic Highly Magnetic Droplets: Magnetic Alignment vs. Viscous Drag
}

\author{
O. Sandre ${ }^{\dagger^{\mathrm{i}}}$, J. Browaeys ${ }^{\dagger}$, R. Perzynski ${ }^{\dagger}$, J-C. Bacri ${ }^{\dagger}$, V. Cabuil ${ }^{\dagger}$, R. E. Rosensweig ${ }^{\dagger}$ \\ $\dagger$ †Lab. Milieux Désordonnés et Hétérogènes, Université P. et M. Curie (Paris 6), Université D. Diderot (Paris 7) UMR \\ 7603 CNRS (Paris 6), Tour 13, Case 78, 75252 Paris Cedex 05, France \\ †ab. Interfaces Ioniques et Chargées, Université P. et M. Curie (Paris 6), UMR 7612 CNRS \\ Bâtiment F, Case 63, 75252 Paris Cedex 05, France
}

\section{$\underline{\text { Abstract }}$}

We report here the collective behavior of droplets in the scale $10^{-6}-10^{-5} \mathrm{~m}$ made of a concentrated magnetic fluid (MF) elongated and oriented by a magnetic field while rotating synchronously with respect to the carrier liquid. Distribution of droplets sizes is studied as a function of a magnetoviscous number $\mathrm{N}_{\mathrm{mv}}$ that quantifies the competition between magnetic field and local vorticity. Liquid state and very low interfacial tension enable both break-up and coalescence processes which are used by the droplets population to reach its dynamic equilibrium. Theoretical analysis of a single drop motion is extended to the case of the drops assembly. Experiments combining rotation and field modulation show an original regime of non-steady rotation in good agreement with theory.

\section{PACS numbers: 47.55.Dz, 47.55.Kf, 75.50.Mm, 83.80.Gv}

Polarizable systems consisting of many elongated objects exhibit interesting dynamic behaviors when submitted to a rotating electric or magnetic field. Similar regimes depending on the angular velocity $\Omega$ of the field are found for mesomorphic molecules in a nematic liquid crystal [1] as well as for macroscopic cylindrical objects made of phospholipidic bilayers [2]. The director axis of the structure rotates synchronously with the field at low $\Omega$ and up to a threshold value $\Omega_{\mathrm{c}}$. Above the threshold, the rotation of the director is not synchronous anymore with the field and its timeaveraged rotation rate is less than $\Omega$. The existence of an upper limit $\Omega_{\mathrm{c}}$ for the angular velocity of the anisotropic objects is due to viscous dissipation in the surrounding liquid. After unlocking with the rotation of the field the objects follow a so called "jerky motion" [3], consisting of a rotation with stops and backward motions. One of the physical parameters that determine the value of $\Omega_{\mathrm{c}}$ is the elongation of the anisotropic objects (more precisely their aspect ratio as will be defined later): the more elongated the objects are, the smaller is the maximum rotation rate $\Omega_{\mathrm{c}}$ they can follow. The interesting feature of the system we present here is that instead of solid objects with given aspect ratio, it is composed of liquid droplets which can exchange matter between themselves [Figs. 1(a), 1(b)]. (a)

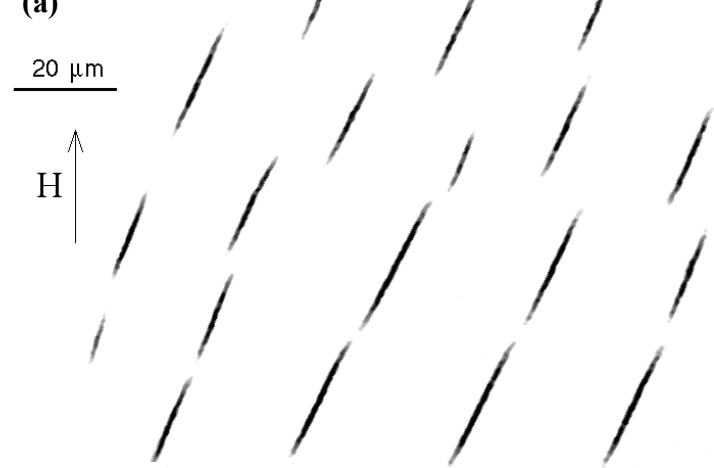

(b)

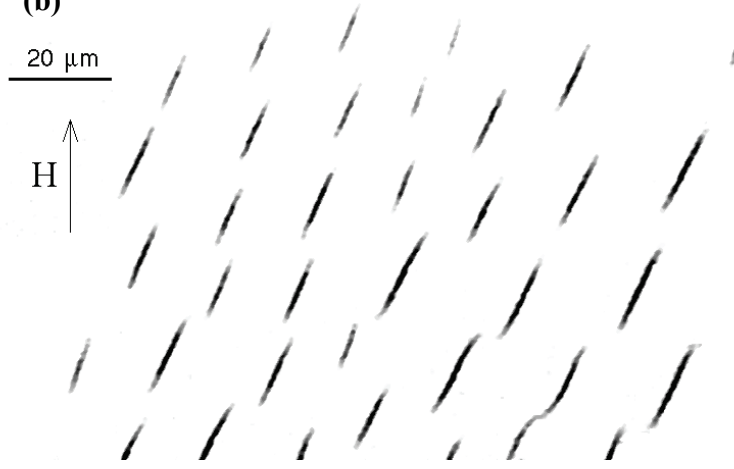

Fig. 1. Instantaneous views of microscopic phase-separated MF droplets with the microscope $(\times 32$ objective) under rotating magnetic field $\mathrm{H}=1270 \mathrm{~A} \cdot \mathrm{m}^{-1}$ for two rotation rates $\Omega / 2 \pi=3.45 \mathrm{~Hz}$ (a) and $\Omega / 2 \pi=5.30 \mathrm{~Hz}$ (b). Corresponding values of the "magneto-viscous" number (see text for definition) are $\mathrm{N}_{\mathrm{mv}}=5 \times 10^{-4}$ (a) and $\mathrm{N}_{\mathrm{mv}}=7 \times 10^{-4}$ (b).

The liquid of those highly magnetic microdroplets is a concentrated suspension of magnetic nanoparticles (volume fraction about 30\%) obtained by "liquid-gas" like transition of a magnetic fluid (MF) [4, 5]. The 
strong deformation of the concentrated phase droplets by a static magnetic field has been studied in detail [6], and the equilibrium shape of droplets is known to be an axisymmetric prolate ellipsoid with aspect ratios above 10. Another previous study describes their behavior under a rotating magnetic field in the regime of high frequency (in the range of $\mathrm{kHz}$ ) where they exhibit shape instabilities called "starfishes" [7].

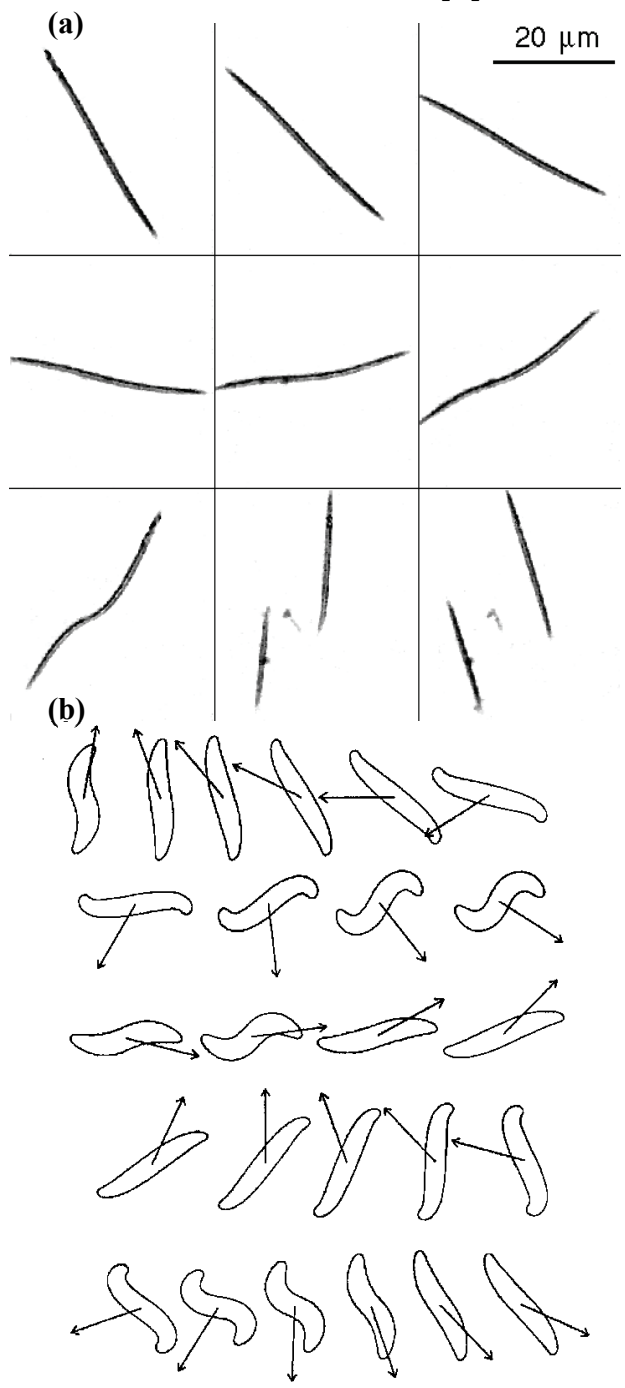

Fig. 2. (a) Experimental observation of drop break-up under rotating field $\left(\Omega=\Omega_{\mathrm{c}}\right.$ ) for magnetic Bond number $\mathrm{B}_{\mathrm{m}}=2.5$ and ratio of inner fluid to outer fluid viscosities $\eta_{i} / \eta_{0}=300$; (b) Numerical simulation of the bending instability just below the onset of break-up computed for $\mathrm{B}_{\mathrm{m}}=105$ and $\eta_{\mathrm{i}} / \eta_{\mathrm{o}}=5$, from [4]. The S-shape occurs when $\vec{H}$ is perpendicular to the droplet central part.

We investigate here a regime of low frequency $(\Omega / 2 \pi<10 \mathrm{~Hz})$ where the droplets remain prolate ellipsoids rotating synchronously with the field but are able to break up to decrease their viscous drag. Above the threshold $\Omega_{\mathrm{c}}$ a droplet divides in several parts with lower aspect ratios (Fig. 2a) which resist further breakup to higher angular velocities. The first steps of the break-up process are well reproduced by numerical simulation [8]: the drop is bent, it develops an "S" shape whose branches usually separate into three smaller droplets [Fig. 2(b)]. To the population of polydisperse liquid droplets is associated a distribution of aspect ratios that adjusts, as we show further on, to minimize the total viscous dissipation. As a result the domain of synchronous rotation is enlarged compared to the case of solid objects. This work is stimulated by previous reports on magneto-rheological effects based on the transfer between mechanical and magnetic energies: "negative viscosity" effect [9] (reduction of effective viscosity by forcing the nanoparticles to rotate faster than the fluid in a shear field flow) and its corollary, magnetovortical resonance [10] (transverse magnetization measured when an oscillating field is applied to a rotating MF). The main difference between those preceding experiments and the new ones is the scale: the rheological effects under magnetic field involve the rotation of the magnetic nanoparticles having diameters around $10 \mathrm{~nm}$, while here we study the rotation of magnetic droplets of a few micrometers. In this paper we investigate two experimental situations: a static MF sample submitted to a rotating magnetic field (case I) and a rotating MF sample under a static magnetic field (case II). After a general theoretical background, we characterize our colloidal system and we describe the experimental setups. The results are then presented and compared to the theoretical predictions.

\section{THEORY}

We propose a simple theory where the droplets are modeled as rotating solid ellipsoids, their liquid state being considered through the variation of their aspect ratios. At first we present the effects of a rotation at $\Omega$ and in a second part we add a simultaneous time modulation of the magnetic field at another pulsation $\omega$. 


\section{A. Rotation at $\Omega$}

The following theory applies indistinctly to the case of droplets rotating synchronously with a magnetic field $\overrightarrow{\mathrm{H}}$ rotating at angular velocity $\Omega$ (case I) or to droplets aligned by a static field in an outer liquid phase submitted to solid rotation at $\Omega$ (case II). The shape of the drops is an axisymmetric prolate ellipsoid with long semi-axis a and short semi-axis b. A rotating magnetic ellipsoid bears on the one hand a magnetic torque $\Gamma_{\mathrm{m}}$ because its long axis is not parallel to $\vec{H}$ and on the other hand a viscous torque $\Gamma_{\mathrm{V}}$ due to its angular velocity relative to the surrounding liquid. The calculation of $\Gamma_{\mathrm{m}}$ is analogous to that of a dielectric ellipsoid tilted at an angle $\theta$ with respect to an electric field [11]:

$$
\Gamma_{\mathrm{m}} / \mathrm{V}=\frac{\chi^{2}(1-3 \mathrm{D})}{2(2+\chi(1-\mathrm{D}))} \mu_{0} \mathrm{H}^{2} \sin (2 \theta)
$$

where $\mathrm{V}, \chi$ and $\mathrm{D}$ are, respectively, the volume, the magnetic susceptibility and the demagnetization factor along the symmetry axis of the ellipsoid. Here $\theta$, which is the angle between the magnetic field and the long axis of the ellipsoid, is positive for $\overrightarrow{\mathrm{H}}$ rotating in advance compared to the droplet. The aspect ratios of the droplets being above 10 , the upper value of $\mathrm{D}$ is 0.02 and hence it will be neglected in the following. The viscous torque $\Gamma_{\mathrm{V}}$ was long ago calculated in the case of a solid ellipsoid of aspect ratio a/b larger than 5 rotating with angular velocity $\frac{\mathrm{d} \theta}{\mathrm{dt}}-\Omega$ relative to the surrounding liquid [12]:

$$
\Gamma_{\mathrm{V}} / \mathrm{V}=\frac{4(\mathrm{a} / \mathrm{b})^{2}}{2 \ln (2 \mathrm{a} / \mathrm{b})-1} \eta_{\mathrm{o}}\left(\frac{\mathrm{d} \theta}{\mathrm{dt}}-\Omega\right)
$$

where $\eta_{0}$ is the viscosity of the outer phase, near that of pure water $\left(10^{-3} \mathrm{~Pa} \cdot \mathrm{s}\right)$. Dynamics of the ellipsoid is governed by the theorem of kinetic momentum:

$$
\mathrm{I} \cdot \frac{\mathrm{d}^{2} \theta}{\mathrm{dt}^{2}}=\Gamma_{\mathrm{m}}+\Gamma_{\mathrm{v}}
$$

where $\mathrm{I}$ is the moment of inertia of the ellipsoid with respect to one of its small semi-axes b. The scaling argument $\mathrm{I} \cdot \frac{\mathrm{d}^{2} \theta}{\mathrm{dt}^{2}} \approx \rho_{\mathrm{i}} \mathrm{Va}^{2} \Omega^{2}, \rho_{\mathrm{i}}$ being the inner phase mass density, and $\Gamma_{\mathrm{V}} \approx \mathrm{V}(\mathrm{a} / \mathrm{b})^{2} \eta_{\mathrm{o}} \Omega$ shows that inertia is negligible as long as $\Omega<<\eta_{\mathrm{o}} / \rho_{\mathrm{i}} \mathrm{b}^{2}$. Using $\rho_{\mathrm{i}}=\phi_{\mathrm{i}} \rho\left(\mathrm{Fe}_{2} \mathrm{O}_{3}\right)+\left(1-\phi_{\mathrm{i}}\right) \rho\left(\mathrm{H}_{2} \mathrm{O}\right) \approx 2 \times 10^{3} \quad \mathrm{~kg} \cdot \mathrm{m}^{-3}$ and $\mathrm{b} \approx 10^{-6} \mathrm{~m}$, one finds $\Omega<<5 \times 10^{5} \mathrm{rad} \cdot \mathrm{s}^{-1}$. The regime presented here corresponds to $\Omega<10^{2} \mathrm{rad} \cdot \mathrm{s}^{-1}$ and thus it is overdamped: after a short transition time, $\theta$ stabilizes at a constant value for which the two torques balance exactly. The condition $\Gamma_{\mathrm{m}}+\Gamma_{\mathrm{V}}=0$ is expressed as

$$
\begin{gathered}
\sin (2 \theta)=\Omega / \Omega_{\mathrm{c}} \\
\text { with } \Omega_{\mathrm{c}}=\frac{\chi^{2}}{2+\chi} \times \frac{2 \ln (2 \mathrm{a} / \mathrm{b})-1}{8(\mathrm{a} / \mathrm{b})^{2}} \mu_{0} \mathrm{H}^{2} / \eta_{\mathrm{o}}
\end{gathered}
$$

to emphasize that the maximum value of the phase lag $\theta$ is $45^{\circ}$ and that the highest angular velocity the ellipsoid can resist, is $\Omega_{\mathrm{c}}$.

The first question about how to adapt the model to the case of rotating liquid droplets is whether formula (2) is still valid for a liquid ellipsoid. The existence of a hydrodynamic flow inside a rotating ellipsoid was studied in detail [13]: it results in a reduction of the viscous torque compared to formula (2) which is significant for a ratio of inner fluid to outer fluid viscosities $\eta_{i} / \eta_{0}$ lower than 10 and for a small eccentricity $e=\sqrt{1-(b / a)^{2}}$ of the ellipsoid. The related experiment was made with a single oily MF drop of initial diameter a few millimeters in an aqueous solution with $\eta_{\mathrm{i}} / \eta_{\mathrm{o}} \approx 5$. In the case of the phase-separated MF the ratio is $\eta_{\mathrm{i}} / \eta_{\mathrm{o}} \approx 3 \times 10^{2}$ (see second part: characterization of the colloid) and the droplets always exhibit e $>0.99$. That is why they behave as solid ellipsoids for the calculation of viscous dissipation. Nevertheless, their liquid state manifests itself through the break-up and coalescence processes, and hence through the distribution of aspect ratio $\mathrm{a} / \mathrm{b}$. We introduce the "magnetoviscous" number as the ratio

$$
\mathrm{N}_{\mathrm{mv}}=\eta_{\mathrm{o}} \Omega / \mu_{0} \chi \mathrm{H}^{2}
$$

that compares the viscous torque to the magnetic one. While $\sin (2 \theta)$ varies linearly with $\mathrm{N}_{\mathrm{mv}}$ for a rigid ellipsoid (with $\chi>>2$ ), it is not true for the system of polydisperse liquid droplets whose mean value of aspect ratio $\mathrm{a} / \mathrm{b}$ is also a function of $\mathrm{N}_{\mathrm{mv}}$. Neglecting the slow logarithmic variation in formula (4), one finds that 
$\sin (2 \theta)=\Omega / \Omega_{\mathrm{c}} \propto(\mathrm{a} / \mathrm{b})^{2} \mathrm{~N}_{\mathrm{mv}} . \quad$ Approximating the variations of the mean aspect ratio by the following scaling law $a / b \propto N_{m v}(v-1) / 2$, we obtain the phenomenological expression $\sin (2 \theta) \propto\left(\mathrm{N}_{\mathrm{mv}}\right)^{\nu}$ given by experiment (see Sec. IV: results). Because raising $\mathrm{N}_{\mathrm{mv}}$ produces both an increase of $\sin (2 \theta)$ and a decrease of a/b through breakup of droplets, $v$ necessarily lies between 0 and 1 . Then the adjustment of the simple model of a rotating ellipsoid to the case of liquid droplets only needs a phenomenological exponent $v$ to take into account their fluidity.

\section{B. Rotation at $\Omega$ and field modulated at $\omega$}

We consider the case of a rotation of the magnetic field with an angular velocity $\Omega$ and a simultaneous sinusoidal modulation, at pulsation $\omega$, of the magnetic field amplitude. Its intensity $\|\overrightarrow{\mathrm{H}}\|$ is thus modulated at

$2 \omega$. Qualitatively the behavior of the droplets is the same as for a rotating field of constant intensity (they rotate synchronously with a phase lag $\theta$ ), except for during the short time when $\|\overrightarrow{\mathrm{H}}\|$ goes through zero. The droplets are then still elongated but no more magnetized, the viscous torque $\Gamma_{\mathrm{V}}$ being the only one to act on them. Hence they follow the motion of the surrounding liquid. For case I they stay stationary in the laboratory coordinates, and for case II they rotate with the outer phase (see, for example, Fig. 9a for case I and Fig. 10 for case II in Sec. IV: results). If $\Gamma_{\mathrm{V}}$ is sufficient the angle $\theta$ between $\overrightarrow{\mathrm{H}}$ and the drop long axis makes periodical sudden turns of $180^{\circ}$ each period of $\|\overrightarrow{\mathrm{H}}\|$ : the resulting motion is a non-steady rotation at pulsation $\omega$. Otherwise $\theta$ only oscillates around a mean position. Those two regimes can be reproduced quantitatively by studying the torques balance equation in time-dependent conditions, taking into account that $\overrightarrow{\mathrm{H}}$ is modulated at pulsation $\omega$. We consider two cases depending on the value of $\omega \tau_{\mathrm{S}}$ where $\tau_{\mathrm{S}}$ is the relaxation time of droplets shape when the field is switched off.

Case i): $\omega \tau_{S}>>2 \pi$. This case implies that the droplets remain elongated during the whole period of
$\|\overrightarrow{\mathrm{H}}\|$ and $\mathrm{a} / \mathrm{b}$ is taken as a constant in following equation:

$$
\begin{aligned}
& \frac{4(\mathrm{a} / \mathrm{b})^{2}}{2 \ln (2 \mathrm{a} / \mathrm{b})-1} \eta_{\mathrm{o}}\left(\Omega-\frac{\mathrm{d} \theta}{\mathrm{dt}}\right) \\
= & \frac{\chi^{2}}{2(2+\chi)} \mu_{0} \mathrm{H}^{2}[\sin (\omega \mathrm{t})]^{2} \sin [2 \theta(\mathrm{t})]
\end{aligned}
$$

It can be rewritten as

$$
\frac{\mathrm{d} \theta}{\mathrm{dt}}=\Omega-\Omega_{\mathrm{c}}[\sin (\omega \mathrm{t})]^{2} \sin [2 \theta(\mathrm{t})],
$$

where $\Omega_{\mathrm{c}}$ is given by formula (4). It is a first order nonlinear differential equation that cannot be solved analytically. The right side is periodic in time and in angle with the respective periods $\pi / \omega$ and $\pi$. Reduced variables $\tau=\omega t / \pi$ and $\alpha=\theta / \pi$ are introduced, so that intervals $\tau \in[0 ; 1]$ and $\alpha \in[0 ; 1]$ represent, respectively, a full period of $\|\overrightarrow{\mathrm{H}}\|$ (thus a half-period of $\overrightarrow{\mathrm{H}}$ ) and a half-turn of the long axis relatively to $\overrightarrow{\mathrm{H}}$. Final rescaling of the equation is obtained with the parameters $\rho=\Omega / \omega$ and $\lambda=\Omega_{\mathrm{c}} / \omega$ :

$$
\mathrm{d} \alpha / \mathrm{d} \tau=\rho-\lambda[\sin (\pi \tau)]^{2} \sin (2 \pi \alpha)
$$

Case ii): $\omega \tau_{\mathrm{S}}<<2 \pi$. In this case the droplet shape has enough time to relax during the decrease of $\|\overrightarrow{\mathrm{H}}\|$. At any time $t$, the aspect ratio $(a / b) t$ has the value that would give a rotation under a field of constant intensity $\|\overrightarrow{\mathrm{H}}\| \mathrm{t}$. It is thus in a "quasi-static" regime. Inferring the same power law $(\mathrm{a} / \mathrm{b})_{\mathrm{t}} \propto\left(\mathrm{N}_{\mathrm{mv}}\right) \mathrm{t}^{(\mathrm{v}-1) / 2}$ as in the simple rotation case (without field modulation), we obtain $(\mathrm{a} / \mathrm{b}) \mathrm{t}^{2} \propto[\sin (\omega \mathrm{t})]^{2(1-v)}$. To take into account this scalng law, $\Omega_{\mathrm{c}}$ has to be replaced by $\Omega_{\mathrm{c}}[\sin (\omega \mathrm{t})]^{2(v-1)}$ in the time-dependent equation (7). In terms of reduced variables $\alpha$ and $\tau$, Eq. (7) then rewrites as

$$
\mathrm{d} \alpha / \mathrm{d} \tau=\rho-\lambda[\sin (\pi \tau)]^{2 \nu} \sin (2 \pi \alpha) .
$$

This expression is general. It includes case i) with $v=1$, meaning that the aspect ratio $a / b$ is constant in time. Numerical simulations of Eq. (9) are performed using the simple Euler method with time step $\Delta \tau=0.001$ and for different values of the parameters $\rho, \lambda$ and $v$. Note that the physical limit restricts the study by the condition $\rho<\lambda$, because the droplets breakup when $\Omega$ goes beyond $\Omega_{\mathrm{c}}$. In all the simulations a permanent regime is reached after a transition time which usually 
do not exceed one period in $\tau$ units. The solutions are never chaotic, which is not surprising as a consequence of the Peixoto theorem [14] for a space of phases $(\alpha ; \tau)$ with a torus surface topology (i.e., $\alpha$ and $\tau$ are both periodic).

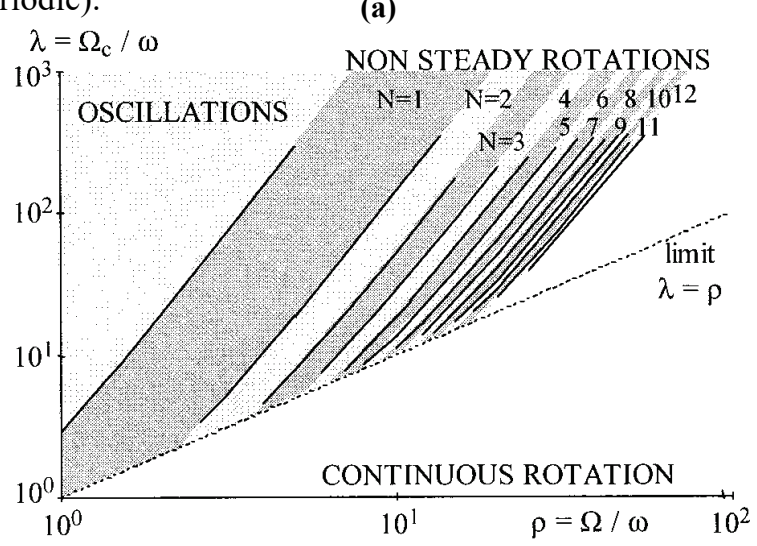

(b)

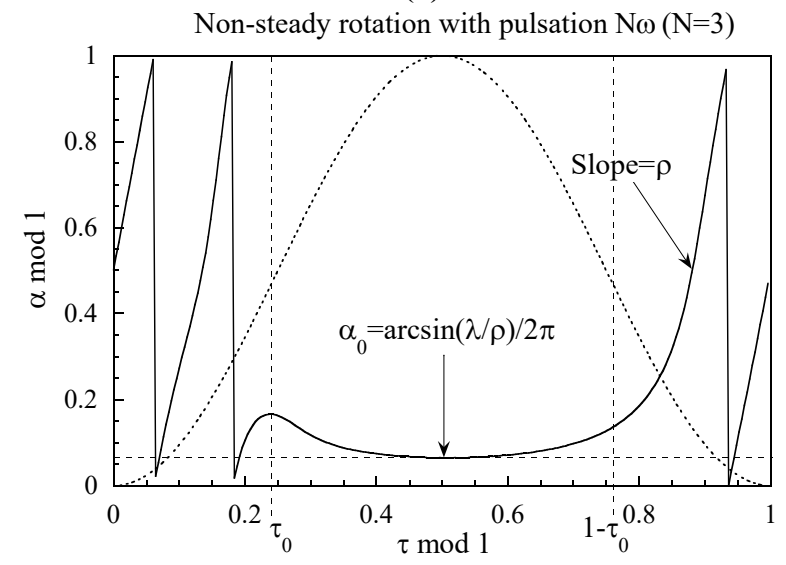

Fig. 3. (a) Phase diagram in plane $(\rho ; \lambda)$ showing the different regimes for a rotating droplet submitted to field modulation in the case of constant aspect ratio (rigid ellipsoid or $\omega \tau_{S}>>2 \pi$ ), from numerical simulation of equation (7). Border lines between the domains are quite parallel with common slope $2.8 \pm 0.1$; (b) Typical solution of tilt angle between field and drop long axis as a function of time in a regime of non-steady rotation with time-averaged pulsation $\mathrm{N} \omega$ (here $\mathrm{N}=3$ ). Solid line is a simulation of equation (7) with $\rho=8$ and $\lambda=20$. Dotted line represents the modulation of the magnetic torque $[\sin (\omega t)]^{2}$.

It is convenient to present the different regimes observed on phase diagrams in the half-plane $\rho<\lambda$. In this theoretical section we show the diagram corresponding to simulations of Eq. (9) with $v=1$ [Fig. 3(a)]. Later in the paper we present the case $v=0.4$ (Fig. 12), which is more appropriate to fit our results. The upper left part of the diagram is the domain of oscillations without rotation (high field and low drag).
The lower right part of the diagram is far below the line $\lambda=\rho$; the solutions tend to the unphysical limit case of continuous rotation $\alpha=\rho \tau$ (this regime is of poor physical meaning for without field aspect ratio is 1 and $\alpha$ is undetermined). The stripes in between are the domains of non-steady rotations with time-averaged pulsation $\mathrm{N} \omega, \mathrm{N}$ being an integer. Their boundaries are quite parallel, with a slope $2.8 \pm 0.1$ for $v=1$ and $1.7 \pm 0.1$ for $v=0.4$. We shall see below that the slopes are identical and equal to $2 v+1$ in a first approximation. Qualitatively it is possible to distinguish to different parts in the solution $\alpha(\tau)$ during the reduced time period $[0 ; 1]$ (see Fig. $3 b$ for an example). In the intervals [0; $\left.\tau_{0}\right]$ and $\left[1-\tau_{0} ; 1\right]$, the angle (in $\alpha$ units) rotates at reduced angular velocity $\mathrm{d} \alpha / \mathrm{d} \tau \approx \rho$. In the interval $\left[\tau_{0} ; 1-\right.$ $\left.\tau_{0}\right]$ it remains almost constant and comparable to the tilt angle obtained for a magnetic field of constant intensity: $\alpha 0=\arcsin (\rho / \lambda) / 2 \pi$. In the conditions of Fig. $3 b \quad(v=1$, $\rho=8, \lambda=20$ ) for example, $\tau_{0} \approx 0.25$ and $\alpha_{0} \approx 0.065$. The crossover between the two behaviors can be defined by $(\mathrm{d} \alpha / \mathrm{d} \tau)\left(\tau=\tau_{0}\right)=0$. However, in a fist approximation we keep $\alpha\left(\tau_{0}\right)$ still equal to $\rho \tau_{0} \quad(\bmod 1)$. Hence linearization of Eq. (9) leads to

$$
\rho-\lambda\left(\pi \tau_{0}\right)^{2 v}\left(2 \pi \rho \tau_{0}\right)=0 \text { so that } \pi \tau_{0}=(2 \lambda)^{-1 /(2 \nu+1)} \text {. }
$$

A non-steady rotation of time-averaged pulsation $\mathrm{N} \omega$ is only possible if the duration of continuous rotation at velocity $\rho$ is $2 \tau_{0}>\mathrm{N} / \rho$, leading to

$$
2 \lambda<\left(\frac{2 \rho}{\pi \mathrm{N}}\right)^{2 v+1}
$$

This condition expresses why, in Fig. 3(a), explains the boundaries between the different regimes of different $\mathrm{N}$ have a slope nearly equal to $2 v+1$. However, only numerical simulations of Eq. (9) give the exact prefactors of the boundaries.

\section{SYNTHESIS AND CHARACTERISTICS \\ A. Colloid}

The use of a phase-separated MF is ideally suited to obtain droplets that can be stretched and oriented by a magnetic field of low intensity. First, from a magnetic point of view, the ratio of the droplet permeability $\mu_{i}$ to 
the permeability $\mu_{\mathrm{O}}$ of the outer phase is more than one order of magnitude higher than for a standard MF drop suspended in immiscible oil. In addition the interfacial tension between the two phases is four orders of magnitude lower.

The first steps of synthesis to get a monophasic aqueous MF are described for example in [15]. It is a dispersion in water of maghemite nanoparticles $(\gamma-$ $\mathrm{Fe}_{2} \mathrm{O}_{3}$ ) bearing superficial charges; thus electrostatic repulsions insure the stability of the dispersion. The particle diameter D roughly follows a log-normal distribution $\quad P(D)=\frac{1}{\sqrt{2 \pi} \sigma D} \exp \left(-\ln ^{2}\left(D / D_{0}\right) / 2 \sigma^{2}\right)$. The polydispersity can be reduced by a size-sorting method [16] down to $\sigma=0.1$. However we use here on the contrary a MF with a broad distribution of diameters: $\mathrm{D}_{0}=6.8 \mathrm{~nm}$ and $\sigma=0.5$ (as deduced from magnetic measurements [5]). Hence relatively large particles are present which favors phase separation. A first order "liquid-gas" like transition is obtained by adding tetramethylammonium hydroxide (TAMOH) to the monophasic MF. This electrolyte screens the electrostatic repulsions, and above an ionic strength threshold a phase separation occurs leading to micronsized droplets of a phase rich in particles in suspension in another phase poor in particles [17]. A given amount concentrated phase is isolated by magnetic sedimentation for characterization. Iron titration gives a volume fraction of particles inside the concentrated phase $\phi_{\mathrm{i}}=31 \%$. Magnetization $\mathrm{M}$ of this concentrated phase is measured as a function of an applied magnetic field $\mathrm{H}_{0}$ [18]. The flux induced by the vibration of the magnetic sample is detected in a coil at the vibrating frequency. The magnetization curve [Fig. 4(a)] is drawn as a function of the internal magnetic field $\mathrm{H}=\mathrm{H}_{0}-\mathrm{D}_{\mathrm{S}} \mathrm{M}$ after correction of the demagnetization effect due to the rod-shaped container holding the strongly magnetic sample. The aspect ratio of the container being equal to 5, we take $\mathrm{DS}_{\mathrm{S}}=0.04$ [19].

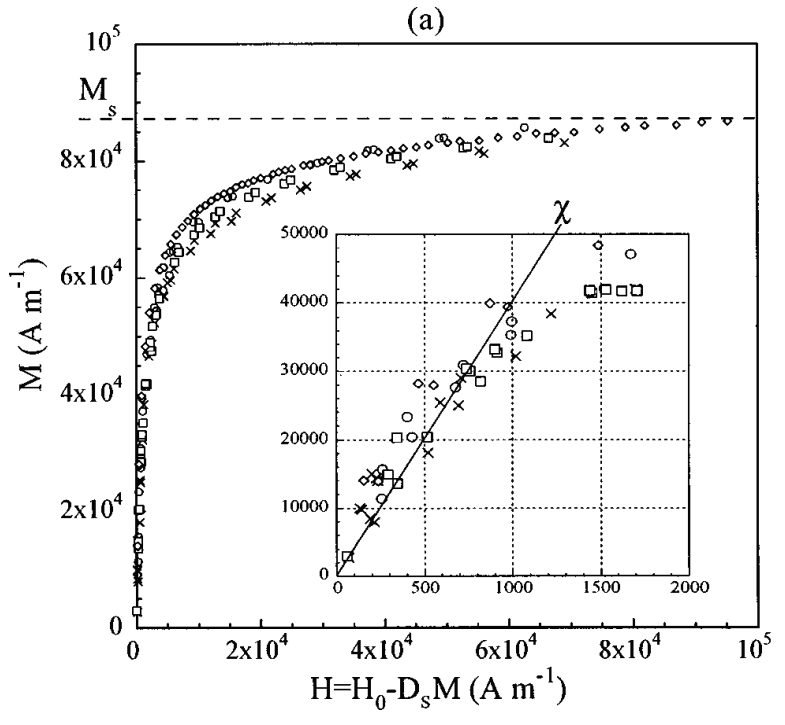

(b)

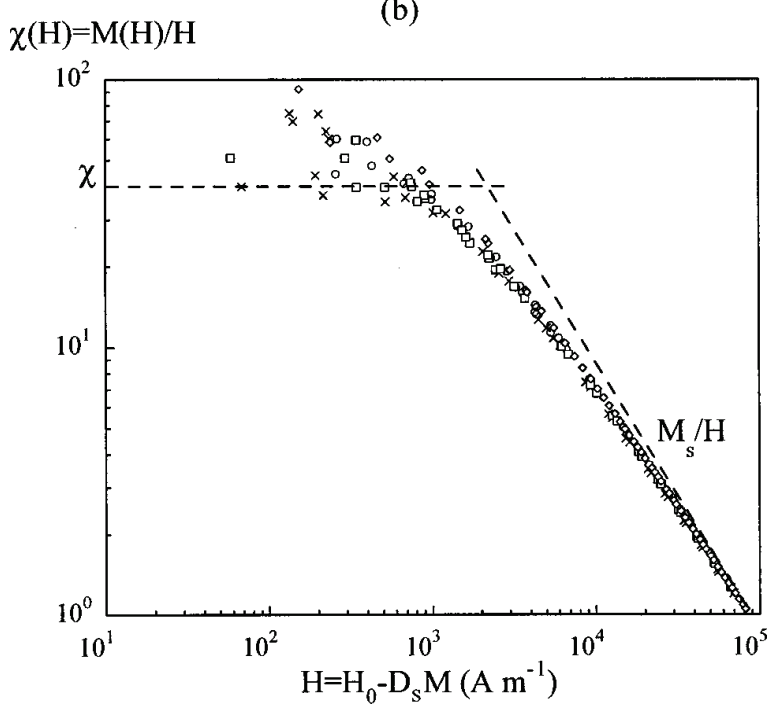

Fig. 4. (a) Magnetization curve $M$ of the pure concentrated phase drawn as a function of the internal magnetic field $\mathrm{H}$, after correction of the demagnetizing effect (sample tube shape corresponds to $\mathrm{DS}_{\mathrm{S}}=0.04$ ). Four cycles of the magnetic field $\mathrm{H}_{0}$ have been applied independently. The slope in the domain of linearity (see magnification in inset) gives the magnetic susceptibility $\chi=\lim _{\mathrm{H} \rightarrow 0} \frac{\mathrm{M}}{\mathrm{H}}=40 \pm 10$. The curve saturates at the value $\mathrm{MS}_{\mathrm{S}}=8.6 \times 10^{4} \mathrm{~A} \cdot \mathrm{m}^{-1}$. (b) Effective susceptibility $[\chi(H)=M(H) / H]$. In the linear regime of $M(H)$, the values are very dispersed around the initial $\chi$ because of the uncertainty of very low values of the magnetic field.

The saturation magnetization of the solution is $\mathrm{MS}_{\mathrm{S}}=8.6 \times 10^{4} \mathrm{~A} \cdot \mathrm{m}^{-1}$. It allows another evaluation of $\phi_{\mathrm{i}}$ from $\mathrm{MS}=\phi_{\mathrm{i}} \mathrm{mS}$ where $\mathrm{mS}=2.7 \times 10^{5} \mathrm{~A} \cdot \mathrm{m}^{-1}$ is the magnetization of the ferrimagnetic grain material [18]. This value of $\mathrm{MS}_{\mathrm{S}}$ leads to $\phi_{\mathrm{i}}=32 \%$. The magnetic susceptibility of the concentrated phase is the initial 
slope of the curve $\chi=\lim _{\mathrm{H} \rightarrow 0} \frac{\mathrm{M}}{\mathrm{H}}=40 \pm 10$. This value is very high but the linearity domain is restricted to magnetic fields below $10^{3} \mathrm{~A} \cdot \mathrm{m}^{-1}$ (12 Oe in cgs system). An effective susceptibility is defined as $\chi(\mathrm{H})=[\mathrm{M}(\mathrm{H}) / \mathrm{H}]$. Figure $4 \mathrm{~b}$ shows that above the domain where $\mathrm{M}(\mathrm{H})$ is linear, $\chi(\mathrm{H})$ is a decreasing function. In spite of its high volume fraction in magnetic nanoparticles $\left(\phi_{\mathrm{i}}=31 \%\right)$, the concentrated phase remains liquid. Its viscosity is evaluated by measuring the relaxation time of magnetic field induced birefringence: $\tau_{\mathrm{R}}=0.14 \mathrm{~ms}$. This value is related to the Brownian relaxation time of the particles $\tau_{\mathrm{B}}=3 \eta \mathrm{V}_{\mathrm{h}} / \mathrm{kT}$ by $\tau_{\mathrm{R}}=\tau_{\mathrm{B}} / 3$. For a sample of polydispersity degree $\sigma=0.5$, the average hydrodynamic volume $\mathrm{V}_{\mathrm{h}}$ of the particles is roughly 10 times higher than their magnetic core $\mathrm{VP}_{\mathrm{P}}=\pi \mathrm{D}_{0}^{3} / 6$ [20]. Then the viscosity of the concentrated phase is $\eta_{\mathrm{i}} \approx 3 \times 10^{-1} \mathrm{~Pa} \cdot \mathrm{s}(3$ Poise). In contrast the dilute phase, which has a volume fraction $\phi_{0} \approx 1 \%$ in particles, has a viscosity almost unchanged compared to pure water and hence $\eta_{\mathrm{i}} / \eta_{\mathrm{o}} \approx 3 \times 10^{2}$.

\section{B. Droplets}

We report here two experiments that characterize the droplets of concentrated phase ("liquid") when it is in equilibrium with dilute phase ("gas"). In the first one we follow the method described in [6]: a droplet with radius $\mathrm{R}_{0}=6.5 \mu \mathrm{m}$ (when spherical) is observed by optical microscopy under an applied magnetic field of constant direction and intensity. An elongated deformation is obtained for fields of very low intensity [Fig. 5(a)] of the order of $120 \mathrm{~A} \cdot \mathrm{m}^{-1}(1.5 \mathrm{Oe})$. The equilibrium shape of the drop results from the balance between interfacial tension and magnetic pressure $\mu_{0}(\vec{M} \cdot \vec{n})^{2} / 2$ acting on the interface between the inner phase of magnetization $\overrightarrow{\mathrm{M}}$ and the outer phase which is negligibly magnetized [4] ( $\vec{n}$ is a normal unit vector). The measured aspect ratio $\mathrm{a} / \mathrm{b}$ is plotted as a function of the magnetic Bond number $\mathrm{B}_{\mathrm{m}}=\mu_{0} \mathrm{H}^{2} \mathrm{R}_{0} / \sigma$ [Fig. 5(b)]. Above a threshold value of $B_{m}$ the drop experiences an elongational shape instability characterized by a steep jump of its aspect ratio. The S-shaped curve can be derived by a minimization of the total free energy of the drop (magnetic energy plus interfacial energy) with respect to $\mathrm{a} / \mathrm{b}$, assuming an ellipsoidal prolate deformation. Fitting the hysteresis curves from measurements on several drops, with formulas (3) and (4) in Ref [6], leads independently to two parameters. On the one hand the interfacial tension $\sigma=9 \pm 2 \times 10^{-7} \mathrm{~N} \cdot \mathrm{m}^{-1}$ is obtained by translating the curves along the $\mathrm{B}_{\mathrm{m}}$ axis (in logarithmic scale), and, on the other hand the magnetic susceptibility $\chi=75 \pm 10$ comes from the optimization of their shape as a whole.

The interfacial tension is so small because the colloidal system is near the critical point of its phase diagram (ionic strength just above the onset of phase separation). An independent determination of similarly small interface tension in a phase-separated ferrofluid system is found in [21] based on the time constant for a stretched droplet to break up into spherical droplets when the magnetic field is suddenly released. The value of the susceptibility is more than an order of magnitude greater than predicted from the Langevin expression for non-interacting particles $\chi=\mu_{0}\left(\phi_{\mathrm{i}} \mathrm{VPmS}^{2} / 3 \mathrm{kT}\right) \approx 0.4$. This law is still obeyed by the ferrofluids concentrated by solvent evaporation. The particularity of the concentrated phase synthesized here by phase separation is that it contains the larger particles of the initial polydisperse ferrofluid, while the smaller ones remain in the dilute phase [16]. Therefore strong dipolar interactions (proportional to $\mathrm{VP}_{\mathrm{P}}^{2}$ ) could be responsible for the deviation from Langevin's law. The phase-separated droplets reported in [6] already displayed such an enormously big initial susceptibility. One still notes that the susceptibilities deduced with the method described earlier in this subsection $(\chi=75 \pm 10)$ are nearly twice as large as those from the magnetization measurement $(\chi=40 \pm 10)$. The variance is likely due to contamination of the bulk sample used in the magnetization determination (see Sec. II A) by smaller particles that decrease the susceptibility. The 
quantity of concentrated phase needed to measure the macroscopic magnetization (about $0.5 \mathrm{~mL}$ ) is much larger than the total volume of the droplets observed on video. Therefore the concentrated phase isolated by magnetic sedimentation must contain a broader size distribution $\mathrm{P}(\mathrm{d})$ of nanoparticles than the microdroplets that mainly originate from the tail of the distribution. Nevertheless $\chi$ values are compatible with extrapolation of $\chi(\mathrm{H})$ in the decade $10^{1}-10^{2} \mathrm{~A} \cdot \mathrm{m}^{-1}$ which is out of the scope of the magnetization experiment [Fig. 4(b)].

(a)

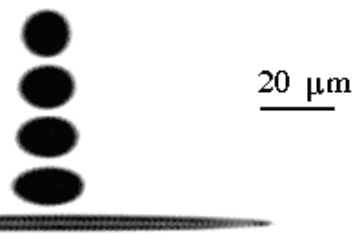

(b)

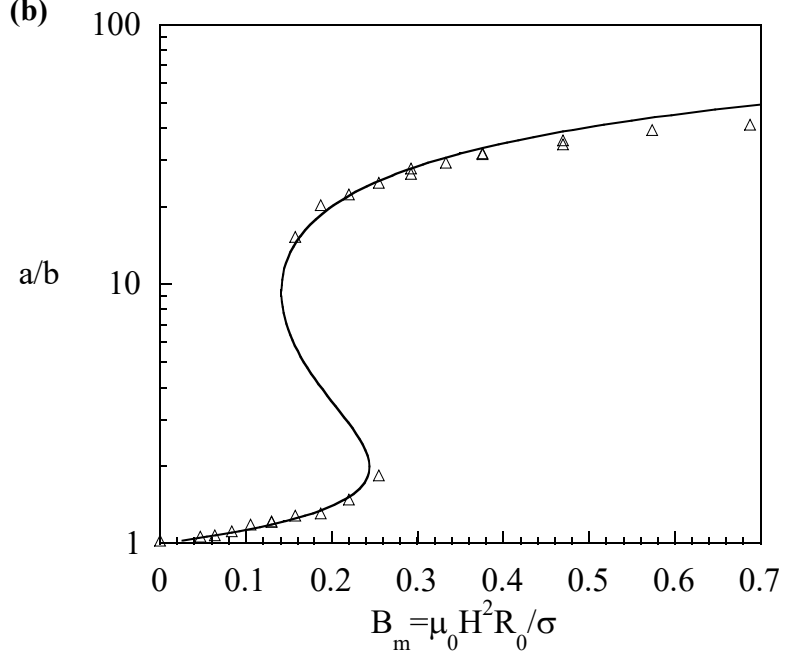

Fig. 5. (a) Drop deformation under static magnetic field observed for increasing values of the field intensity (successively $\mathrm{H}=0,117,138,149,160,182,224$ and 320 $A \cdot \mathrm{m}^{-1}$ ). Initial shape of the drop is spherical with radius $\mathrm{R}_{0}=6.5 \mu \mathrm{m}$; (b) Aspect ratio $\mathrm{a} / \mathrm{b}$ as a function of $\mathrm{B}_{\mathrm{m}}$ and corresponding fit giving $\sigma=7.5 \times 10^{-7} \mathrm{~N} \cdot \mathrm{m}^{-1}$ and $\chi=63$.

The second experiment is the measurement of the characteristic time $\tau_{\mathrm{S}}$ taken by a droplet to relax its shape after switching off the field. A previous work on dynamics of phase separated MF droplets concerned the time necessary to establish the elongational shape instability [22]. The diverging of this time on approaching the critical value of $\mathrm{B}_{\mathrm{m}}$ was in full agreement with a theoretical model. We are interested here in the reverse mechanism of shape relaxation. The following experimental relaxation law $\mathrm{a} /\left.\mathrm{b}\right|_{\mathrm{t}}-1=\mathrm{a} /\left.\mathrm{b}\right|_{\mathrm{t}=0}$ $-1] \exp \left(-\mathrm{t} / \tau_{\mathrm{S}}\right)$ is verified using several droplets with different final radius $\mathrm{R}_{0}$ between 1.5 and $2.5 \mu \mathrm{m}$. It gives a mean value $\tau \mathrm{S}=0.2 \mathrm{~s}$, which is in agreement with the rough estimation from scaling arguments: $\tau_{\mathrm{S}} \approx \mathrm{R}_{0} \eta_{\mathrm{i}} / \sigma=0.6 \mathrm{~s}\left(\mathrm{R}_{0}=2 \mu \mathrm{m}, \eta_{\mathrm{i}}=0.3 \mathrm{~Pa} \cdot \mathrm{s}\right.$ and $\sigma=10^{-6}$ $\mathrm{N} \cdot \mathrm{m}^{-1}$ ). We deduce that the droplets under a magnetic field oscillating at pulsation $\omega$ remain in the "quasistatic" regime (case ii) as long as $\omega / 2 \pi<\tau \mathrm{S}^{-1}=5 \mathrm{~Hz}$.

\section{EXPERIMENTAL SETUPS}

To both equivalent situations of either droplets rotating synchronously with $\overrightarrow{\mathrm{H}}$ at angular velocity $\Omega$ (case I) or droplets aligned by a static field in a carrier liquid prone to solid rotation at $\Omega$ (case II) correspond two different setups called, respectively, I and II:

In setup I the droplets of concentrated phase are observed under a microscope (objective with $32 \times$ magnification) while rotating the magnetic field $\vec{H}$; circular polarization of $\overrightarrow{\mathrm{H}}$ is obtained with two pairs of coils oriented at $90^{\circ}$ and supplied by ac currents of frequency $\mathrm{f}=\Omega / 2 \pi$ in phase quadrature [Fig. 6(a)]; the instantaneous direction of $\overrightarrow{\mathrm{H}}$ is known thanks to a light pulse (LP1), of time width $4 \mathrm{~ms}$, triggered with one of its components;

In setup II the magnetic field has a constant direction while the sample of phase-separated MF is rotated [Fig. 6(b)]; for that purpose the sample cell is set at the end of a hollow tube rotated at constant speed $\Omega$; a laser beam parallel to $\vec{\Omega}$ and normal to $\vec{H}$ travels through the tube and is diffracted by the sample; the resulting pattern is observed on a screen.

Because both methods need optical transparency, the sample is a quartz cell of thickness $100 \mu \mathrm{m}$ filled with phase-separated MF. The pictures (direct observation of droplets for I or scattering patterns for II) are filmed with a charge-coupled device (CCD) camera connected to a tape recorder and digitized on a computer. The video system samples 50 pictures per second and a 
shutter integrates each picture for only $4 \mathrm{~ms}$ instead of $20 \mathrm{~ms}$ to improve the image sharpness. In that condition the number of sequences is sufficient to follow rotation of droplets [Fig. 9(a)] or of scattering pattern (Fig. 10) up to $\Omega / 2 \pi=6 \mathrm{~Hz}$. The tilt angle $\theta$ between field and droplets long axes is measured: In setup I from the phase lag between rotating field and rotating droplets (field phase is given by the light pulse LP1); in setup II from the tilt angle of the scattering pattern with respect to its direction for zero rotation.
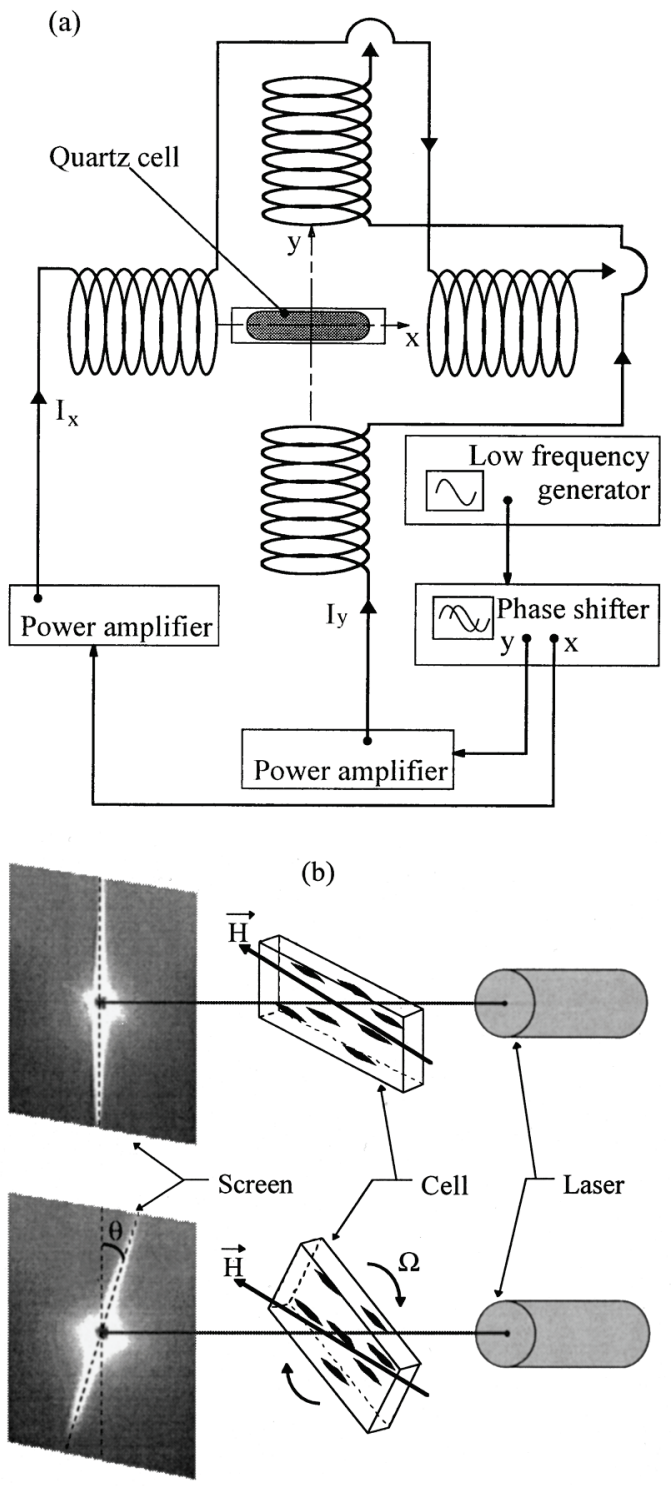

Fig. 6. (a) Sketch of setup I to produce a rotating magnetic field in a quartz cell filled with phase-separated MF observed under a microscope; this method enables to follow singles droplets independently; (b) sketch of setup II to obtain a solid rotation of the cell under a field of constant direction; the scattering pattern by the laser beam becomes tilted at an angle $\theta$ representing the phase lag between droplets long axis and $\vec{H}$ averaged on a large amount of droplets.
The way to modulate the field is different in the two experiments. For the direct observations (setup I), the components of $\overrightarrow{\mathrm{H}}$,

$$
\left\{\begin{array}{l}
H_{x}(t)=H \sin (\omega t) \cos (\Omega t) \\
H_{y}(t)=H \sin (\omega t) \sin (\Omega t)
\end{array}\right.
$$

are created by two numerical signal generators (two DSN105-60MHz cards, VDATA, France) driven by a PC computer. In the scattering experiment (setup II), which is in $\overrightarrow{\mathrm{H}}$ coordinates, a field oscillating at pulsation $\omega$ is simply applied to the sample rotating at angular velocity $\Omega$. In both setups, a light pulse LP2 of time width $4 \mathrm{~ms}$ is triggered with $\overrightarrow{\mathrm{H}}$ to know its phase.

\section{RESULTS}

\section{A. Rotation at $\Omega$}

Setup I (direct observation of rotating droplets). From direct observation on pictures similar to those of Figs. 1(a) and 1(b) it is clear that the more elongated the droplets are, the higher is their phase lag $\theta$. This setup enables to study the statistical distribution of aspect ratios for different values of angular velocity $\Omega$ and field intensity $H$. The data are averaged on approximately 100 droplets and standard deviation is represented by error bars (Fig. 7).

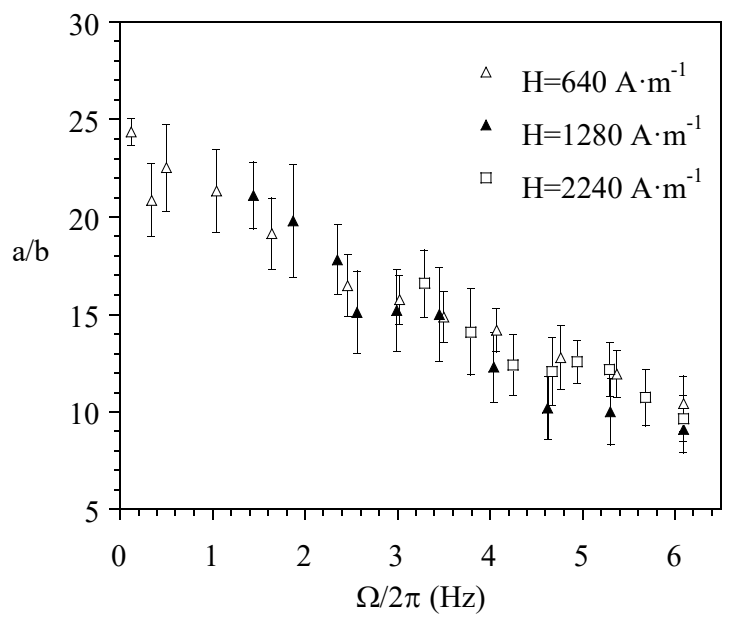

Fig. 7. Mean aspect ratio $\mathrm{a} / \mathrm{b}$ of droplets from measurements with setup I for different values of $H$ and $\Omega$. Each point is averaged on approximately 100 droplets and the error bars stand for the standard deviation.

The mean aspect ratio appears to be a decreasing function of $\Omega$ due to the break-up of droplets. As a consequence an increase of $\Omega$ leads to smaller droplets which are more numerous and become closer to each 
other. Then they tend to form a hexagonal network because of the dynamic repulsive interaction between the rotating droplets. On the contrary reducing $\Omega$ raises back the mean aspect ratio. This perfect reversibility of $a / b$ as a function of $\Omega$ is a consequence of the simultaneous presence of the two antagonist processes of droplets breakup and droplets coalescence.

Setup II (observation of the scattering pattern). This second method is suitable for the study of the influence of $\Omega$ and $H$ on the value of the phase lag $\theta$. It performs an average on a large amount of droplets. Experiments are made by varying $\Omega$ at constant $H$ and data are presented in Fig. 8, where $\sin (2 \theta)$ is plotted as a function of the magnetoviscous number $\mathrm{N}_{\mathrm{mv}}$ [see Eq. (5)]. We observe experimentally that there is a wide range of $\mathrm{N}_{\mathrm{mv}}$ where two scattering lines are observed simultaneously (see enclosed box in Fig. 8). They correspond, respectively, to a small value $\theta_{\mathrm{S}}$ and a large one $\theta_{1}$ of the tilt angle $\theta$. Interestingly, all the data are superposed on a master curve with two branches $\theta_{\mathrm{S}}$ and $\theta_{1}$. These are described over two orders of magnitude by scaling laws $\sin (2 \theta)=\Omega / \Omega_{\mathrm{c}} \propto \mathrm{N}_{\mathrm{mv}}{ }^{v}$ with, respectively, $\nu=0.60 \pm 0.07$ for $\theta_{S}$ and $\nu=0.28 \pm 0.05$ for $\theta_{1}$.

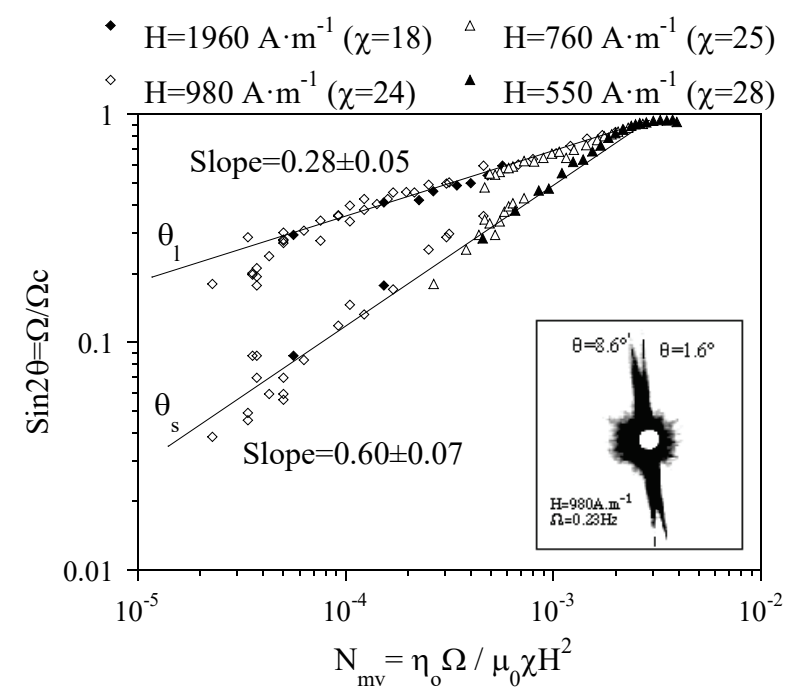

Fig. 8. Experimental data of the tilt angle $\theta$ for different $H$ and $\Omega$ measured with setup II and put together on a master curve with ordinate $\sin 2 \theta$ and abscissa the magnetoviscous number $\mathrm{N}_{\mathrm{mv}}$. The coexistence of a small tilt angle $\theta_{\mathrm{S}}$ and a large one $\theta_{1}$ (see enclosed picture) is attributed to permanent break-up of drops and the two branches of the curve $\theta_{\mathrm{S}}$ and $\theta_{1}$ are fitted by power laws.

\section{B. Rotation at $\Omega$ and field modulated at $\omega$}

Setup I. An example of the behavior of a single droplet submitted to a magnetic field rotating at angular velocity $\Omega$ and oscillating at pulsation $\omega$ is reported on Fig. 9(a) where the sequence is labeled in increasing $\tau$ units. The arrow gives the instantaneous direction of $\overrightarrow{\mathrm{H}}$. In the first and fourth rows of views the phase lag $\theta$ between drop axis and $\overrightarrow{\mathrm{H}}$ is constant: these periods belong to the reduced time interval $\left[\tau_{0} ; 1-\tau_{0}\right]$ using the notation of the theoretical section. The value $\tau_{0} \approx 0.1$ is estimated from Fig. 9(b), where reduced angle $\alpha=\theta / \pi$ is plotted as a function of $\tau$ for the droplet of Fig. 9(a).
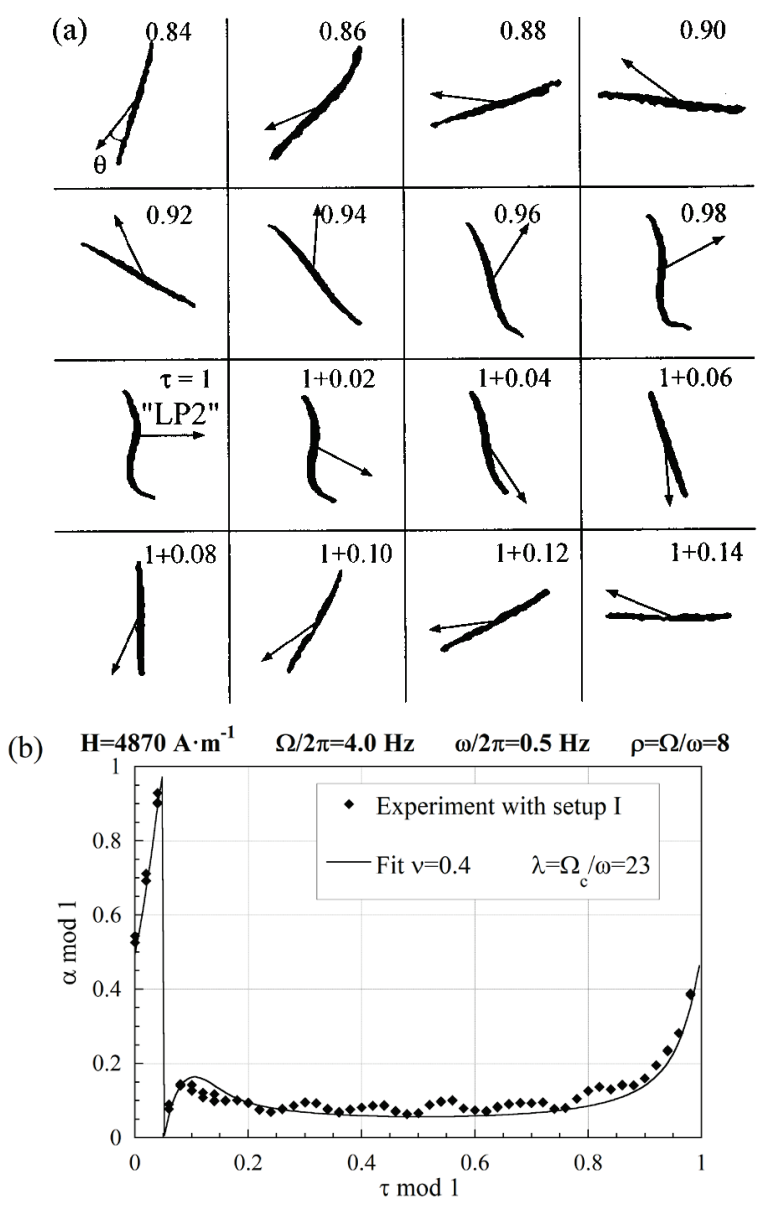

Fig. 9. Regime of nonsteady rotation at pulsation $\omega$ studied with setup I. (a) Single droplet observed when submitted to a field $\overrightarrow{\mathrm{H}}$ rotating clockwise at rate $\Omega / 2 \pi=4 \mathrm{~Hz}$ and modulated at frequency $\omega / 2 \pi=0.5 \mathrm{~Hz}$ (amplitude $\mathrm{H}=4870$ $\mathrm{A} \cdot \mathrm{m}^{-1}$ ). The instantaneous direction of the field is given by an arrow. Time is counted in $\tau$ units (modulo 1). (b) Reduced angle $\alpha$ as a function of reduced time $\tau$ from direct observation of previous droplet with setup I (diamonds) and from numerical solution of Eq. (9) with parameters $v=0.4$, $\rho=\Omega / \omega=8$ and $\lambda=23$ (solid line). 
The two central rows in Fig. 9(a) show the temporary drop stop when $\|\overrightarrow{\mathrm{H}}\|$ is going through zero, associated with $\tau<0.1$ and $\tau>0.9$ of Fig. 9(b). The drop being quiet and the field still rotating, a one half-turn phase lag between drop long axis and $\vec{H}$ is obtained during the $\tau$ interval $[\mathrm{n}+0.9 ; \mathrm{n}+1+0.1]$ where $\mathrm{n}$ is an integer. The solid line fitting quiet well the experimental data in Fig. 9(b) comes from numerical simulation of Eq. (9) with parameters $v=0.4$ and $\lambda=23$. One may note, however, I, Fig. 9(a) that during the half-turn (two central row) the drop experiences not only a relaxation of its aspect ratio $a / b$ but also a slight $\mathrm{S}$-shape bending deformation.

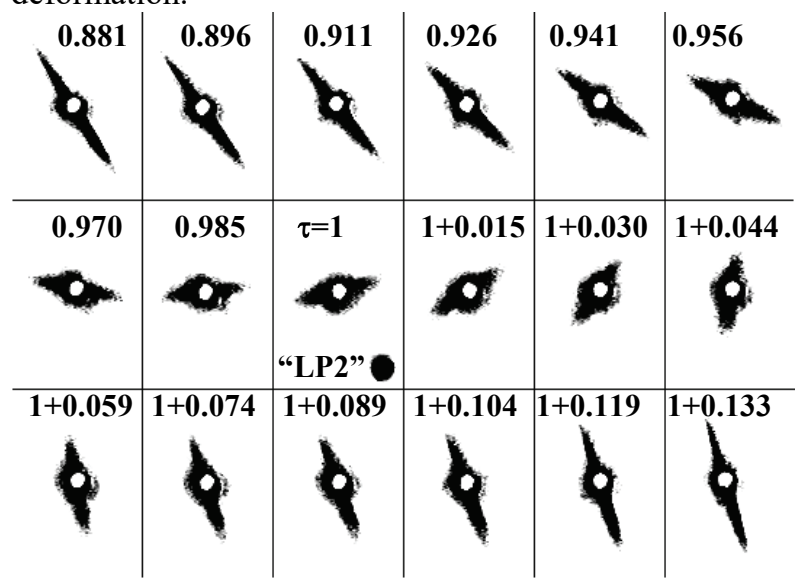

Fig. 10. Regime of nonsteady rotation at pulsation $\omega$ studied with setup II. The sequence shows the half-turn of the scattering pattern when an oscillating field $\left(\mathrm{H}=2000 \mathrm{~A} \cdot \mathrm{m}^{-1}\right.$, $\omega / 2 \pi=0.37 \mathrm{~Hz}$ ) is applied to a population of droplets in a cell rotating counterclockwise $(\Omega / 2 \pi=5 \mathrm{~Hz})$. The angle $\theta$ is measured between scattering pattern axis and vertical direction, and the instant when $\|\overrightarrow{\mathrm{H}}\|=0$ is noticed by the light pulse LP2. Time is counted in $\tau$ units (modulo 1 ).

Setup II. The nonsteady rotation at pulsation $\omega$ of the scattering pattern of a droplets population is the direct visualization of this striking regime. The half-turn of the scattering line occurs every half-period of the field modulation. It is reported on Fig. 10 for given experimental conditions $\left(\mathrm{H}=2000 \quad \mathrm{~A} \cdot \mathrm{m}^{-1}, \Omega / 2 \pi=5\right.$ $\mathrm{Hz}, \omega / 2 \pi=0.37 \mathrm{~Hz}$ ). Simultaneously a thickening of the line is observed and can be attributed to both temporary decrease of droplets aspect ratios and S-shape deformations. The instantaneous position of the scattering line with respect to its direction for zero rotation (i.e. vertical line) is followed precisely as a function of time in various conditions. For the sake of simplicity the field amplitude is kept constant $(\mathrm{H}=2000$ $\mathrm{A} \cdot \mathrm{m}^{-1}$ ) and only the effect of $\Omega$ is studied. Four populations of droplets resulting from four values $\Omega / 2 \pi$ $(2,3,4$, and $5 \mathrm{~Hz})$ of the sample rotation rate are considered. For each population several frequencies of the field modulation $\omega / 2 \pi$ ranging from 0.37 to $5 \mathrm{~Hz}$ are tested. Typical experimental data and their fitting curves by numerical solutions of Eq. (9) are presented in Fig. 11. The most common regimes obtained experimentally are mere oscillations of angle $\alpha$ [Fig. 11(a)] and nonsteady rotation with time-averaged pulsation $\omega$ [Fig. 11(b)]. Nevertheless there are several cases where the scattering pattern is composed of a main line in one regime and a secondary one in another: for example, on both sides of the border between oscillation and rotation [Fig. 11(c)]. Another experiment gives the main line in nonsteady rotation at $\omega$ and the secondary one in nonsteady rotation at $6 \omega$ [Fig. 11(d)].
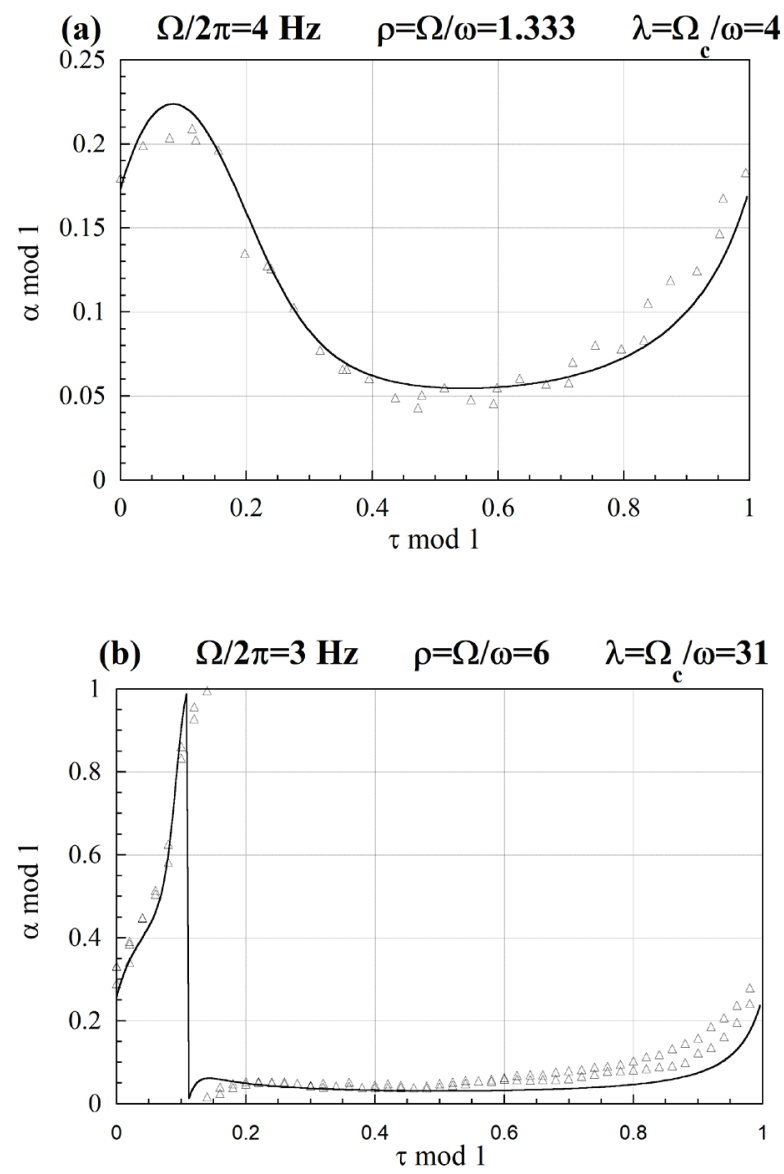

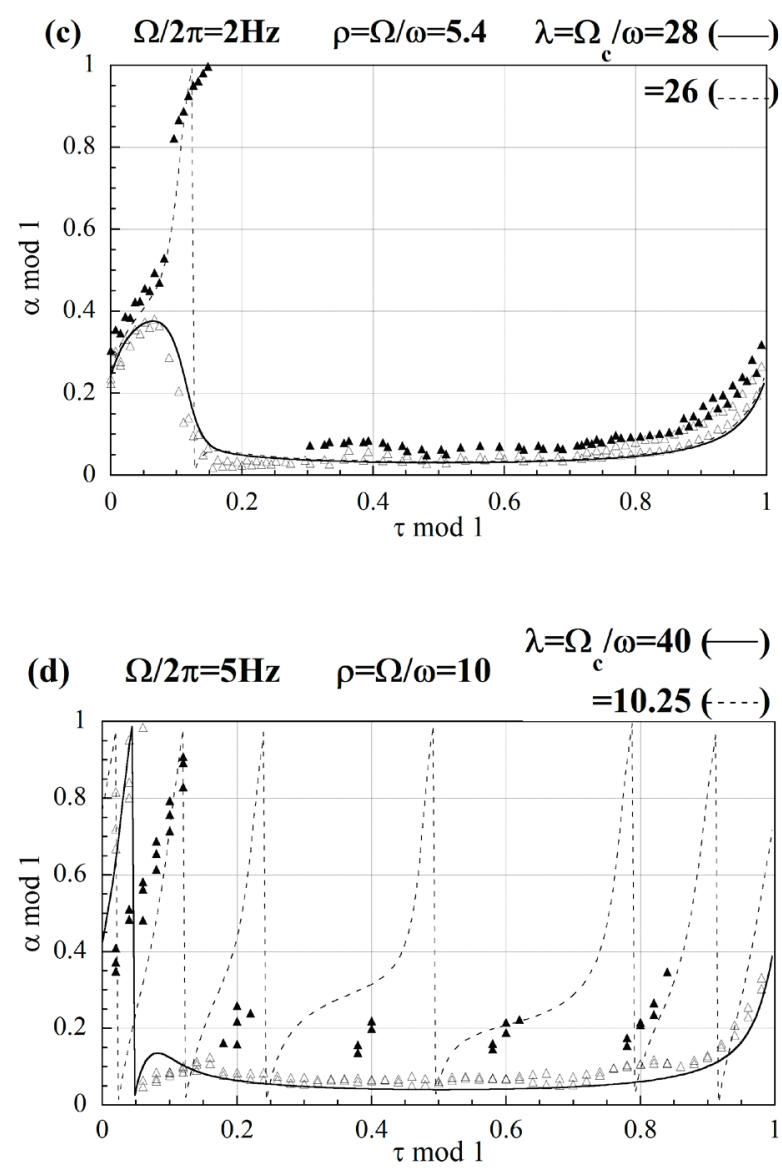

Fig. 11. Different experimental responses obtained with setup II by rotating the cell at angular velocity $\Omega$ and modulating the field of amplitude $\mathrm{H}=2000 \mathrm{~A} \cdot \mathrm{m}^{-1}$ at pulsation $\omega$. The fitting curves are numerical simulations of Eq. (9) where the exponent $v=0.4$ is fixed, $\rho=\Omega / \omega$ is a direct ratio of experimental values and $\lambda=\Omega_{\mathrm{c}} / \omega$ is the only variable parameter. (a) $\Omega / 2 \pi=4 \mathrm{~Hz}, \rho=1.333$ : oscillation $(\lambda=4)$. (b) $\Omega / 2 \pi=3 \mathrm{~Hz}, \rho=6$ : nonsteady rotation with time-averaged pulsation $\omega(\lambda=31)$. (c) $\Omega / 2 \pi=2 \mathrm{~Hz}, \rho=5.4$ : two components on both sides of the border between oscillation $(\lambda=28$, solid line) and non-steady rotation ( $\lambda=26$, dotted line). (d) $\Omega / 2 \pi=5$ $\mathrm{Hz}, \rho=10$ : two components in nonsteady rotations, main line at $\omega(\lambda=40$, solid line $)$ and secondary line at $6 \omega(\lambda=10.25$, dotted line).

Figure 12 regroups all the experiments placed on the phase diagram in plane $(\rho ; \lambda)$. Each marker has an abscissa $\rho=\Omega / \omega$ which is the direct ratio of experimental values. Its ordinate $\lambda=\Omega_{\mathrm{c}} / \omega$ corresponding to the numerical solution of Eq. (9) with $v=0.4$ leading to the best fit of the experimental curve $\alpha(\tau)$. Figure 12 also presents the numerical phase diagram with $v=0.4$ : the stripes which are quite parallel with a common slope $1.7 \pm 0.1$ and decreasing widths are the domains associated to the different regimes of the numerical solutions.

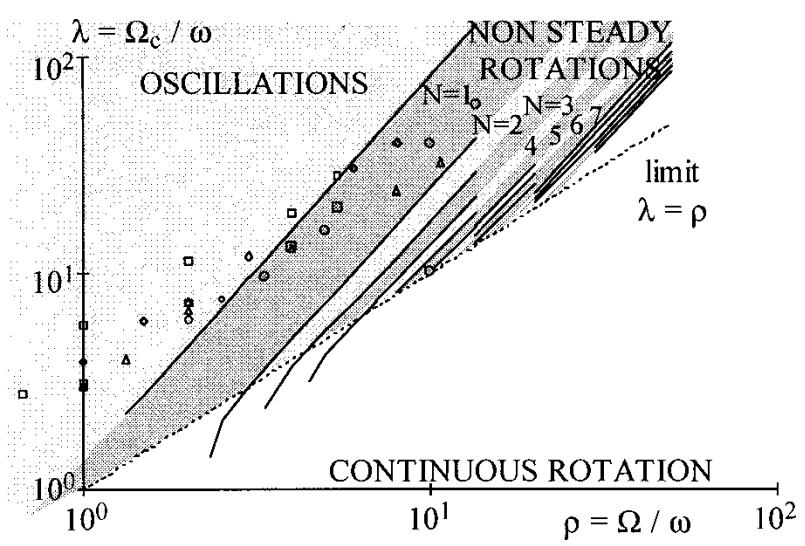

Fig. 12. Phase diagram in plane $(\rho ; \lambda)$ showing the different regimes for a rotating droplet submitted to field modulation in the case of variable aspect ratio (liquid ellipsoid with

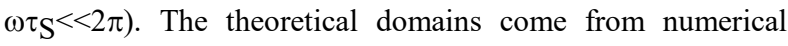
simulation of Eq. (9) with an exponent $v=0.4$ to take into account the shape relaxation. Their border lines are quite parallel with common slope $1.7 \pm 0.1$. The values of $\lambda$ fitting the experimental investigations with setup II at different $\omega$ are placed on the diagram for constant amplitude $\mathrm{H}=2000 \mathrm{~A} \cdot \mathrm{m}^{-1}$ and for several values of $\Omega / 2 \pi$ (squares, $2 \mathrm{~Hz}$; diamonds, 3 $\mathrm{Hz}$; triangles, $4 \mathrm{~Hz}$; and circles, $5 \mathrm{~Hz}$ ). Empty markers stand for oscillation regimes and filled ones for rotations.

\section{DISCUSSION}

\section{A. Rotation at $\Omega$}

We have observed with setup II that the scattering pattern of a population of droplets often consists of two main lines indicating two values $\theta_{\mathrm{S}}$ and $\theta_{1}$ of the tilt angle. In contrast, the droplets aspect ratios measured with setup I are distributed around a unique peak value $\mathrm{a} / \mathrm{b}$. The study of the two-dimensional Fourier transform (FT) of an S-shaped droplet (Fig. 13) provides a simple explanation. As the drop shape may be schematically described by a central segment and two parallel tips [Fig. 2(a)], the FT gives two lines in the perpendicular directions of those three segments. The central part of the " $\mathrm{S}$ " originates in the long axis of the drop before deformation and thus corresponds to the large tilt $\theta_{1}$. 


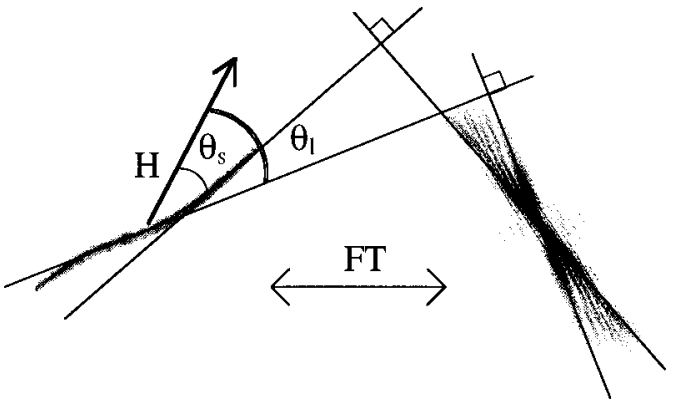

Fig. 13. Two-dimensional Fourier transform of the picture from Fig. 2a showing the droplet bent and developing an Sshape.

The two tips which make a smaller angle $\theta_{\mathrm{S}}$ with respect to the field progressively grow to the detriment of the central segment and will lead to the two daughter droplets after breakup. The permanent coexistence of the two components $\theta_{\mathrm{S}}$ and $\theta_{\mathrm{l}}$ thus indicates that breakup processes continuously occur among the drops. Breakup and recombination processes are indeed observed on video tapes. Then the interpretation for the two branches in Fig. 8 is that the population of droplets consist of the ones that have just broken up (associated with $\theta_{\mathrm{S}}$ ) and the others that still have not (associated with $\theta_{1}$ ). For example most of the droplets of Fig. 1(a), which correspond to $\mathrm{N}_{\mathrm{mv}}=5 \times 10^{-4}$ have larger phaselags than the ones of Fig. 1(b) for which $\mathrm{N}_{\mathrm{mv}}=7 \times 10^{-4}$ because the former is on the $\theta_{1}$ branch while the latter is on the $\theta_{\mathrm{S}}$ one. However, none of the phenomenological laws $\mathrm{a} / \mathrm{b} \propto \mathrm{N}_{\mathrm{mv}}(\mathrm{v}-1) / 2$ deduced from the two experimental laws $\sin (2 \theta) \propto\left(\mathrm{N}_{\mathrm{mv}}\right)^{v}$ for $\theta_{\mathrm{S}}$ and $\theta_{1}$ is verified by direct measurements of $a / b$ with setup I. It could be due to bias induced on the statistics made by direct observation with the microscope of setup I, which is more sensitive to the largest droplets while the scattering pattern in setup II makes an average on the whole population. Nevertheless the reversibility and monotonous decreasing of $\mathrm{a} / \mathrm{b}$ as a function of $\Omega$ (Fig. 7) is the evidence that the coalescence of droplets through attraction between aligned magnetic dipoles always competes with the antagonist process of breakup. The criterion that determines this dynamic system of droplets is very probably the minimization of the total viscous dissipation.

\section{B. Rotation at $\Omega$ and field modulated at $\omega$}

The good fitting of data coming from setup I by numerical simulation [Fig. 9(b)] means that Eq. (9) is rigorously valid for a single droplet of given aspect ratio $a / b$ and hence for a unique value of $\lambda$. The fits are less accurate with the data from experiment II dealing with a polydisperse population of droplets. Nevertheless the model remains a good approximation considering $\lambda$ as the mean value of a distribution. We recall that the shape relaxation of droplets is modeled by a power law of $\mathrm{a} / \mathrm{b}$ as a function of the time dependent field modulus: $\mathrm{a} / \mathrm{b} \propto|\mathrm{H} \sin (\omega \mathrm{t})|^{1-v}$. We point out that the phenomenological exponent $v$ is always held at a constant value $v=0.4$. Taking into account that $\lambda$ is the only adjustable parameter of the fit, the results given by the quasistatic model [case (ii) in Sec. I] are very satisfying. The experiments are made with a field pulsation $\omega$ always below the upper limit of that regime, which is $\omega / 2 \pi=\tau \mathrm{S}^{-1}=5 \mathrm{~Hz}$. Of course there is a simplification in assuming that the droplets always remain ellipsoidal while direct observation with setup I shows that they are temporary deformed into S-shapes.

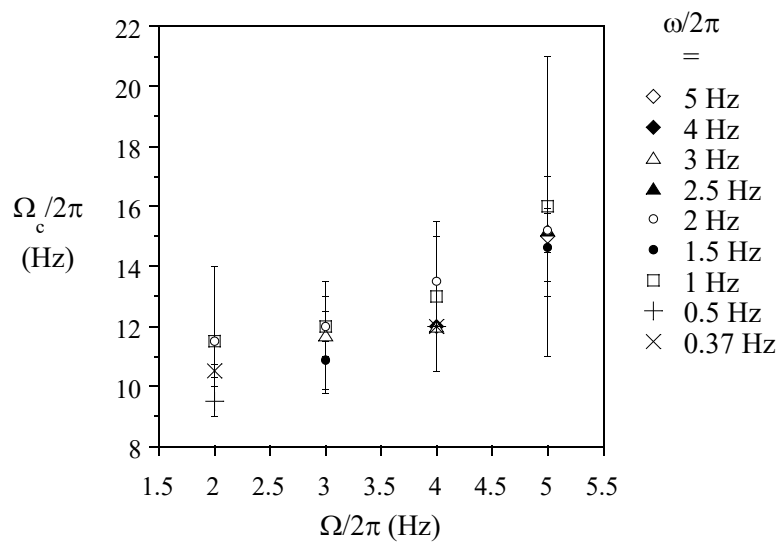

Fig. 14. Mean critical angular velocity $\Omega_{\mathrm{c}}$ of the droplets population as a function of the applied vorticity $\Omega$ deduced from the $\lambda$ parameters used to fit the experimental data $\alpha(\tau)$ obtained with setup II under several field pulsations $\omega$. The error bars come from the uncertainty of the fits.

Finally the physical relevance of the $\lambda$ parameters resulting from the fits is tested by observing their dependence on experimental conditions. Changing the field pulsation $\omega$ (and hence $\rho=\Omega / \omega$ ) at constant $H$ and 
$\Omega$ is a non-disruptive method to investigate a population with a given statistics of aspect ratios and thus a given mean value of $\lambda$ and hence of $\Omega_{\mathrm{c}}$. An estimation of $\Omega_{\mathrm{c}}$ is deduced for each of the four populations with corresponding values of $\Omega / 2 \pi$, respectively, equal to 2 , 3, 4 and $5 \mathrm{~Hz}$ (Fig. 14). The result is that variations of $\Omega_{\mathrm{C}}$ with $\omega$ are negligible compared to the method uncertainty while the increasing of $\Omega_{\mathrm{c}}$ with $\Omega$ is fully established. This is another evidence that the system of liquid droplets adjusts the distribution of aspect ratios through breakup and coalescence processes in response to the applied vorticity $\Omega$.

\section{CONCLUSION}

We have extended the physical problem of polarizable objects rotating in a surrounding fluid to the all liquid case when the two phases are in the liquid state. The main consequence is the ability for the droplets to breakup when they can no longer bear the rotation velocity $\Omega$ due to viscous friction. As a result the regime of synchronous rotation of the structure has a larger domain of angular velocities than for solid objects of same initial size distribution. When modulation of the field intensity at pulsation $\omega$ is superposed to the rotation $\Omega$, a novel regime of nonsteady rotation at averaged velocity $\omega$ is revealed. $A$ priori this regime can also be observed for solid objects and we give a theoretical phase diagram including regimes of non-steady rotations at pulsation $\mathrm{N} \omega$ where $\mathrm{N}$ is an integer. The experimental results for the all liquid system in either presence or absence of field modulation have been reproduced by a simple model in which the fluidity of the droplets is considered through the influence of rotation on the size distribution. The two basic processes of that adaptability are shown to be droplet breakup and droplet coalescence. Their permanent balance is possible because of the extreme softness of the interface between the two liquid phases. This condition is fulfilled by the use of a phaseseparated MF, which unites a huge magnetic polarizability and an extremely low interfacial tension.
Interestingly the concentrated phase of this biphasic material (relative magnetic permeability $\mu_{\mathrm{r}}=75 \pm 10$ ) is analogous for magnetic polarizability to water for electric polarizability (relative dielectric permittivity $\left.\varepsilon_{\mathrm{r}}=78\right)$. Finally the interfacial tension $\left(\sigma \approx 10^{-6} \mathrm{~N} \cdot \mathrm{m}^{-1}\right)$ is the parameter that limits the minimal size of the droplets (by limiting the maximum total surface between the two phases) and gives an upper limit to their angular velocity. Thus it determines the crossover between the regime of synchronous rotation of prolate ellipsoids studied currently and the one of asynchronous rotation of spiky oblate droplets reported in [7].

\section{ACKNOWLEDGMENTS}

This work was supported by the Direction Générale de l'Armement (Grant No. NB 96-1149) and by the Conseil Régional d'île de France, through the "Chaire Blaise Pascal" administered by "La Fondation de l'École Normale Supérieure".

\section{REFERENCES}

[1] F. Brochard, J. Phys. (France) Lett. 35, 19 (1974); F. Brochard, L. Léger, and R. B. Meyer, J. Phys. (Paris) 36, 209 (1975).

[2] D. Lettelier, O. Sandre, C. Ménager, V. Cabuil. and M. Lavergne, J. Mat. Sc. Eng. C 5, 153 (1997).

[3] G. Helgesen, P. Pieranski, and A. T. Skjeltorp, Phys. Rev. Lett. 64, 1425 (1990); Phys. Rev. A 42, 7271 (1990).

[4] R. E. Rosensweig, Ferrohydrodynamics (Dover, New-York, 1997)

[5] Magnetic Fluids and Applications Handbook, edited by B. Berkovski (Begell House, New York, 1996).

[6] J-C. Bacri, D. Salin, J. Phys. Lett. 43 L-649 (1982).

[7] J-C. Bacri, A. Cebers, and R. Perzynski, Phys. Rev. Lett. 72(17), 2705 (1994).

[8] S. Lacis, PhD Thesis, University Paris VII, 1996.

[9] J-C. Bacri, R. Perzynski, M. I. Shliomis, and G. I. Burde, Phys. Rev. Lett. 75(11), 2128 (1995); R. E. Rosensweig, Science 271, 614 (1996); F. Gazeau, C. Baravian, J.-C. Bacri, R. Perzynski, and M. Shliomis, Phys. Rev. E 56, 614 (1997).

[10] F. Gazeau, B. M. Heegaard, J.-C. Bacri, A. Cebers, and R. Perzynski, Europhys. Lett. 35(8), 609 (1996).

[11] L. Landau, E. Lifchitz, Electrodynamique des milieux continus (Mir, Moscow, 1969) p. 67.

[12] J. Perrin, J. Phys. Radium 7(5) 497 (1934).

[13] A. V. Lebedev, K. I. Morozov, Pis'ma Zh. Eksp. Teor. Fiz. 65, 150 (1997) [JETP Lett. 65, 161 (1997)].

[14] P. Manneville, Dissipative structures and weak turbulence (Academic Press, New York 1990).

[15] S. Lefebure, PhD thesis, University Paris VI, 1996. 
[16] R. Massart, E. Dubois, V. Cabuil, and E. Hasmonay, J. Magn. Magn. Mater. 149, 1 (1995).

[17] J-C. Bacri, D. Salin, R. Perzynski, V. Cabuil, and R. Massart, J. Colloid Interface Sci. 132, 43 (1989).

[18] J-C. Bacri, D. Salin, R. Perzynski, V. Cabuil, and R. Massart, J. Magn. Magn. Mater. 62, 36 (1986).

[19] S. Chikazumi, Physics of Magnetism, $2^{\text {nd }}$ ed. (Wiley, New York, 1966), p. 22.

[20] J-C. Bacri, R. Perzynski, D. Salin, and J. Servais, J. Phys. (Paris) 48, 1385 (1987).

[21] R. E. Rosensweig, and J. Popplewell, Electromagnetic Forces and Applications, edited by J. Tani and T. Takagi (Elsevier Science, New-York, 1992), pp 83-86.

[22] J-C. Bacri, D. Salin, J. Phys. (France) Lett. 44 L415 (1983). 